\title{
Sous une critique de la criminologie, une critique des rationalités pénales
}

Beyond a critic of criminology : a critic of the rationale for penalties

\section{Olivier Razac et Fabien Gouriou}

\section{(2) OpenEdition \\ 1 Journals}

\section{Édition électronique}

URL : http://journals.openedition.org/conflits/18919

DOI : 10.4000/conflits. 18919

ISSN : $1777-5345$

Éditeur :

CCLS - Centre d'études sur les conflits lilberté et sécurité, L'Harmattan

\section{Édition imprimée}

Date de publication : 31 décembre 2014

Pagination : 225-240

ISBN : 978-2-343-05760-6

ISSN : 1157-996X

Référence électronique

Olivier Razac et Fabien Gouriou, "Sous une critique de la criminologie, une critique des rationalités pénales », Cultures \& Conflits [En ligne], 94-95-96 | été-automne-hiver 2014, mis en ligne le 20 février 2016, consulté le 30 mars 2021. URL : http://journals.openedition.org/conflits/18919; DOI : https:// doi.org/10.4000/conflits. 18919 


\section{Sous une critique de la criminologie, une critique des rationalités pénales}

\section{Olivier RAZAC, Fabien GOURIOU}

Olivier Razac est philosophe, Maître de conférences en philosophie à l'Université Pierre-Mendès-France, Grenoble.

Fabien Gouriou est docteur en psychologie, travailleur indépendant et chercheur associé au Centre Interdisciplinaire d'Analyse des Processus Humains et Sociaux (CIAPHS), Université Rennes 2.

$\mathrm{I}$ serait difficile de trouver aujourd'hui un ouvrage se réclamant de près ou de loin de ce qui se nomme "criminologie » qui ne cite pas, au moins, Surveiller et punir ${ }^{1}$. Or, cette évidence devrait pourtant surprendre si l'on constate, naïvement, que Foucault parle certes de prison, de criminalité, de psychiatrie criminelle... mais si peu de « criminologie ». Ceci peut s'expliquer par plusieurs raisons. D'abord, au moment où Foucault travaille sur le champ pénal, il n'y a pas en France une discipline universitaire criminologique constituée, comme c'est alors le cas, par exemple, en Belgique ou au Canada 2. Ensuite, les réformes judiciaires, pénitentiaires, les dispositifs concrets et les pratiques des professionnels ne font que très exceptionnellement référence à un savoir criminologique (ceci malgré le mouvement de la défense sociale nouvelle en France). Enfin, et surtout, Foucault ne se donne pas pour objet la critique d'une forme de savoir qui serait la criminologie ; pas plus d'ailleurs qu'il ne le fait pour la psychiatrie ou la médecine.

La conférence inaugurale du cours dispensé en 1981 à l'Université catholique de Louvain est à ce titre fort explicite : "Je n'ai pas essayé de savoir si le discours des psychiatres était vrai, ni celui des médecins, bien que ce problème soit tout à fait légitime ; je n'ai pas essayé de déterminer à quelle idéologie obéissait le discours des criminologues - bien que ce soit également un pro-

1. Foucault M., Surveiller et punir, Paris, Gallimard, 1995 [1975].

2. L'École de criminologie de Louvain - ou plus exactement l'École des Sciences criminelles de Louvain - est fondée par Louis Braffort en 1929. C'est en partie sur son modèle que Denis Szabo fondera en 1960 l’École de criminologie de Montréal. 
blème intéressant. Le problème que j'ai voulu poser était différent : c'était de m'interroger sur les raisons et les formes de l'entreprise de dire vrai à propos de choses comme la folie, la maladie ou le crime ${ }^{3}$ ".

Prononcé à l'invitation de l'École de criminologie, le cours est guidé par la question du gouvernement par la vérité, saisi dans les rapports noués entre juridiction et véridiction dans la pratique pénale à travers la forme particulière de l'aveu. Ici la critique ne porte précisément pas sur la «criminologie » comme telle (terme dont le lecteur peinera d'ailleurs à trouver au fil des pages plus de cinq, ou six, occurrences), mais sur l'émergence dans le droit pénal moderne d'une subjectivité criminelle dont la consistance tient à un singulier déplacement de la fonction de l'aveu. Il ne s'agit plus d'avouer le crime pour lequel on est jugé mais de dire la vérité de son être-même ; mode d'énonciation projetant désormais le crime dans une trame subjective supposée signifiante. Du crime au criminel, de l'acte à la dangerosité, de la punition du coupable à la protection de la société... Dans cette série d'opérations, où le deuxième terme ne remplace pas mais se superpose au premier, la juridiction du juge est nécessairement doublée d'autre chose : «Il faut un savoir, il faut qu'il y ait à propos du sujet et de sa vérité un type de connaissance, un type de savoir, un type d'expérience, un type aussi d'échange et de dialogue qui ne pouvait relever que de la psychiatrie, la criminologie, la psychologie 4 ».

Comme il l'expliquera quelques temps plus tard, ce que Foucault cherche à comprendre, c'est « le système de rationalité sous-jacent aux pratiques punitives » car, dans le sillage nietzschéen de la Généalogie de la morale, " tout se passe comme si nous pratiquions une punition en laissant valoir, sédimentées un peu les unes sur les autres, un certain nombre d'idées hétérogènes, qui relèvent d'histoires différentes, de moments distincts, de rationalités divergentes ${ }^{5}$ ». Dans ce cadre, c'est moins un savoir sur le crime qui attire spécialement l'attention, qu'une série de dispositifs plus concrets entrelaçant l'exercice d'un pouvoir et la prétention d'un savoir, au premier chef desquels l'expertise psychiatrique pénale. C'est en effet à cet endroit que les enjeux, aussi bien épistémologiques que politiques, sont tangibles, en tant qu'un pouvoir judiciaire et un savoir médical tous deux en crise de légitimité trouvent dans le branchement de l'un sur l'autre l'occasion rêvée, et historiquement situable, d'un renforcement mutuel et d'une extension de leur emprise. C'est très précisément ce chiasme, cet enroulement, qui constitue le fond de la critique foucaldienne.

3. Foucault M., Mal faire, dire vrai. Fonction de l'aven en justice. Cours de Louvain, 1981, Louvain, Presses universitaires de Louvain / Chicago, University of Chicago Press, 2012, pp. 9-10.

4. Ibid., p. 223.

5. Foucault M., "Qu’appelle-t-on punir ? ", in Foucault M., Dits et écrits, Tome IV, Paris, Gallimard, 1994 [1984], pp. 636-646, p. 637. 
Or, on pourrait croire que les choses ont profondément changé depuis. La progressive consolidation académique de la criminologie en serait un premier marqueur même si, d'un pays à l'autre, sa place et donc sa reconnaissance dans le paysage universitaire diffère tout de même notablement. Second marqueur, son influence de plus en plus explicite dans le champ pénal, en l'occurrence dans les pratiques pénitentiaires qui seront présentement l'objet de notre analyse. De telles évolutions pourraient laisser suggérer que la dispersion initiale de la nébuleuse criminologique a désormais cédé la place à un dispositif à la fois autonome et localisé : « la » criminologie comme corps doctrinal constitué, qu'un frayage foucaldien s'emploierait alors à déconstruire comme tel. Pourtant, si les choses semblent changer, le problème initial persiste. D'un côté, si l'on pose qu'un "savoir » criminologique existe, force est d'admettre la persistance de son éclatement tant épistémologique qu'institutionnel, solidaire d'une légitimité bien fragile ${ }^{6}$. De l'autre, l'évidence de son ancrage dans les pratiques pénitentiaires est à la mesure de son ambiguïté, comme nous allons tâcher de l'expliciter. De telle manière que si l'on veut faire la critique d'une criminologie institutionnalisée ou celle d'un tournant criminologique des pratiques pénitentiaires, on a bien souvent l'impression de brasser du vent, en particulier en France.

D’où cette proposition méthodologique : il ne s'agit pas de partir d'un prisme criminologique pour analyser la rationalité des pratiques, mais de partir du montage de rationalités dont témoignent les pratiques pour y repérer la place et le rôle d'une rationalité criminologique. Ceci suppose tout d'abord une analyse discursive visant à cartographier les différentes rationalités qui structurent le champ pénitentiaire actuel (I), afin de clarifier le système de relations qu'elles entretiennent les unes avec les autres (II). Cette cartographie doit permettre de saisir comment une rationalité criminologique s'insère dans cette configuration et en redistribue le jeu de relations (III). C'est à partir d'une telle analyse que les enjeux épistémologiques et politiques d'une « criminologie » pourront dès lors être saisis : elle n'est pas une « nouvelle » rationalité de la peine qui se substituerait pas à pas à une autre, mais l'une des pièces d'un montage éclectique de rationalités qui permet de faire fonctionner un nouvel arbitraire de la justice.

Cet article repose sur une recherche menée entre 2011 et 2013 intitulée Les rationalités de la probation française ${ }^{7}$. C'est pourquoi nous limiterons notre propos à l'activité des Services pénitentiaires d'insertion et de probation

6. En témoigne la «polémique » française autour de la création d'une chaire universitaire de criminologie. Sur ce sujet, voir par exemple : Mucchielli L., "Vers une criminologie d'État en France ? Institutions, acteurs et doctrine d'une nouvelle science policière. ", Politix, 89-1, 2010, pp. 195-214 et, de l'autre côté, Bauer A., Raufer X., Roucaute Y., «Une vocation nouvelle pour la criminologie », Sécurité globale, Automne 2008.

7. Razac O. (CIRAP/ENAP), Gouriou F. (CIAPHS) et Salle G. (CLERSÉ), Les rationalités de la probation française, Rapport de recherche, Cirap/Enap, Ministère de la Justice, 2013. Source internet : http://www.enap.justice.fr/recherche/actualite.php?actu=386 
(SPIP) pour ce qui concerne les mesures hors détention, donc en milieu dit «ouvert ». Considérant par ailleurs que, d'une part, la question de l'influence ou de l'usage de la criminologie y est plus vivace qu'en milieu dit «fermé » et que, d'autre part, la configuration de rationalités y est exemplaire d'un éclectisme légitimant des pratiques tendanciellement arbitraires de gouvernementalité.

\section{Les rationalités de la probation française}

Avant de proposer une cartographie des rationalités structurant le champ pénitentiaire de la probation, il faut définir ce que l'on entend ici par « rationalité ». Le niveau d'analyse choisi s'inspire des indications méthodologiques qui parsèment l'œuvre de Foucault - de l'Archéologie du savoir 8 aux derniers textes - et selon lesquelles il faut partir de la discursivité, de la pluralité et de la contingence de la rationalité. La raison ne doit pas être conçue comme un universel apodictique et substantialisé mais comme un jeu entre des formations de discours possédant une certaine consistance interne. "Ce que j’ai essayé d'analyser, ce sont des formes de rationalité : différentes instaurations, différentes créations, différentes modifications par lesquelles des rationalités s'engendrent les unes les autres, s'opposent les unes aux autres, se chassent les unes les autres, sans que pour autant on puisse assigner un moment où on serait passé de la rationalité à l'irrationnalité ${ }^{9}$. » Ces formes de rationalité se saisissent à un niveau discursif, c'est-à-dire au niveau des choses effectivement dites, "énoncées ", et de la structure qu'elles manifestent. La consistance minimale d'une rationalité se trouve dans la liaison entre une finalité spécifique et une série de moyens conçus comme nécessaires pour réaliser cet objectif. La première chose à analyser dans une institution est ainsi «sa rationalité ou sa fin, c'est-à-dire les objectifs qu'elle se propose et les moyens dont elle dispose pour atteindre ces objectifs. C'est en somme le programme de l'institution 10 ». Mais, pour compléter cette définition, il est éclairant de considérer aussi les places ou positions logiquement impliquées pour celui qui met en œuvre cette rationalité (position de sujet) et celui sur qui elle s'exerce (position d'objet).

Or, le champ pénitentiaire, et celui de la probation en particulier, ne livre pas spontanément des formations de discours ayant atteint le niveau minimum de consistance pour Foucault, c'est-à-dire un seuil de positivité tel qu'une pratique discursive individualisée fasse fonctionner un seul système de formation des énoncés. Les recherches sur ce champ et ses professionnels insistent au contraire sur l'éclatement des missions ${ }^{11}$, la fragilité identitaire ${ }^{12}$ ou la disper-

8. Foucault M., L'archéologie du savoir, Paris, Gallimard, 1969.

9. Foucault M., "Structuralisme et poststructuralisme », in Foucault M., Dits et écrits, Tome IV, Paris, Gallimard, 1994 [1983], pp. 431-457, p. 441.

10. Foucault M., «Qu'appelle-t-on punir ?», op. cit., p. 639.

11. Chauvenet A., Orlic F., "Sens de la peine et contraintes en milieu ouvert et fermé », Déviance et société, 26-4, 2002, pp. 443-461. 
sion et l'artisanat des pratiques ${ }^{13}$. D’où la nécessité d'un premier repérage généalogique afin d'effectuer le « découpage provisoire » de "régions initiales » de rationalité ${ }^{14}$. Selon cette méthode, des grands ensembles - euxmêmes complexes, évolutifs, perméables - sont aisément repérables.

La probation française repose, en premier lieu, sur un triptyque de logiques constitutives. Dès la création des services sociaux des prisons (1945) et ensuite des Comités de probation et d'assistance aux libérés (CPAL, 1958), qui deviendront ensuite les Services pénitentiaires d'insertion et de probation (SPIP, 1999), on trouve trois logiques structurantes : les assistantes sociales, les éducateurs pénitentiaires et les bénévoles ont un rôle éducatif compris selon un prisme moral, et un rôle social compris selon un prisme solidaire et humaniste, le tout pour des citoyens condamnés par la loi pénale ${ }^{15}$. Cette action socio-éducative sous mandat pénal constitue toujours le socle sur lequel s'appuient les pratiques de probation en France. Il est en lui-même complexe, ces trois rationalités (pénale, éducative, sociale) n’étant pas nécessairement harmonieuses, mais les débats en sont relativement connus et stabilisés. Or, trois autres rationalités ont pris une importance croissante dans l'application des peines en venant singulièrement complexifier le sens, opérationnel et symbolique, des pratiques.

Tout d'abord, une rationalité sanitaire reconfigure l'application des peines, en particulier à travers le recours toujours plus massif aux différentes formes de soins pénalement ordonnés : obligation de soins ${ }^{16}$, injonction thérapeutique ${ }^{17}$ et injonction de soins ${ }^{18}$. Ce mouvement a été nettement favorisé par une «pathologisation » de la délinquance autour des figures du toxicomane et du délinquant sexuel et, plus globalement, des addictions et des violences. Ensuite, une rationalité de gestion des risques criminels, dont nous verrons que c'est à elle qu'il faudrait réserver le terme de « criminologie » si l'on souhaite retrouver un peu de clarté dans l'analyse des enjeux de la pénalité. S’il n'existe pas en France une science institutionnalisée d'évaluation et de traitement des risques criminels, il est manifeste que des formes de discours dits «criminologiques » produisent des effets tangibles sur la pratique des SPIP. Auparavant marginal, le terme est devenu central dans les textes normatifs

12. Lhuilier D. (ed.), Changements et construction des identités professionnelles : les travailleurs sociaux pénitentiaires, Paris, Direction de l'administration pénitentiaire, juillet 2007.

13. Dindo S., Sursis avec mise à l'épreuve : la peine méconnue. Une analyse des pratiques de probation en France, Paris, Direction de l'administration pénitentiaire/Bureau PMJ 1, mai 2011.

14. Foucault M., L'archéologie du savoir, op. cit., p. 42.

15. Faugeron C., Le Boulaire J.-M., «La création du service social des prisons et l'évolution de la réforme pénitentiaire en France de 1945 à 1958 », Déviance et société, 12-4, 1988, pp. 317-359, p. 39.

16. Loi de 1954 sur le traitement des alcooliques dangereux, puis la création en 1958 du sursis avec mise à l'épreuve.

17. Loi de 1970 relative à la lutte contre la toxicomanie.

18. Loi de 1998 relative à la prévention et à la répression des infractions sexuelles, instituant le suivi socio-judiciaire. 
récents. La circulaire du 19 mars 2008 relative aux missions et aux méthodes d'intervention des SPIP 19 subordonne ainsi l'action des services à la mission de « prévention de la récidive ", précisant que cette mission doit être comprise selon une «dimension criminologique » et une «dimension sociale ». La même circulaire fait aussi référence aux « connaissances en criminologie » sur lesquelles les agents sont enjoints à s'appuyer dans leur action, et ces orientations se concrétisent dans la production de dispositifs tels que le diagnostic à visée criminologique (DAVC) et les programmes de prévention de la récidive (PPR). Enfin, ces cinq logiques possédant chacune leurs exigences en termes de prise en charge des personnes sont en relation avec une logique plus organisationnelle de «nouvelle gestion publique », au sens d'une transposition de « l'esprit gestionnaire » et managérial auparavant réservé au firmes privées concurrentielles ${ }^{20}$, dont les signes manifestes ont été la loi organique relative aux lois de finances (LOLF) promulguée le 1er août 2001 et la Révision générale des politiques publiques (RGPP) en 2008.

Pour autant, ce premier repérage ne suffit pas à cartographier le système de rationalités de la probation française car il reste trop superficiel. Nous n'y reconnaissons que la manière avec laquelle on peut nommer des ensembles discursifs juxtaposés et informant les pratiques mais nous n'atteignons pas leur consistance logique propre, ce qui permettrait de les identifier comme des rationalités distinctes les unes des autres et dont on pourrait alors décrire le système de relations. De plus, ce repérage peut être particulièrement trompeur, car rien ne garantit la réalité de ce découpage. L'éducatif est par exemple un «monde » en soi, ayant intégré des références psychanalytiques, des considérations sociales, de même qu'il reste indissociable des enjeux de gestion des risques et pris dans les difficultés de gouvernementalité néolibérale. Et ceci est vrai pour toutes les autres logiques, dont la porosité est si grande que leur individualisation pourrait finalement n'être que rhétorique : l'éducatif se nommerait parfois criminologique, le sanitaire participerait de la gestion des risques, le pénal aurait des vertus éducatives, la réinsertion sociale préviendrait la récidive, etc. C'est pourquoi il nous faut faire un effort de distinction pour fixer ces rationalités par ce qui les caractérise en propre, et à l'exception de ce qui caractérise les autres. Si l'on trouve une définition minimale qui en constitue un noyau dur, absent des autres logiques, on se donne alors les moyens de comprendre le fonctionnement du système de rationalités à partir duquel les pratiques s'orientent et se légitiment. À l'inverse, si l'on accepte un relâchement sémantique tel qu'il permette de dire, par exemple, que toute action est thérapeutique et qu'elle participe à la prévention de la récidive tout en améliorant la qualité du service public pénitentiaire pour les condamnés usagers, on se prive de toute possibilité de problématiser l'éclectisme pénal actuel et ses

19. Circulaire DAP n 113/PMJ1 du 19 mars 2008, relative aux missions et aux méthodes d'intervention des services pénitentiaires d'insertion et de probation.

20. Ogien A., L'esprit gestionnaire. Une analyse de l'air du temps, Paris, Éditions de l'EHESS, 1996. 
effets de pouvoir. D'où cette nouvelle proposition d'un repérage structural des rationalités qui permette d'isoler méthodologiquement leurs logiques propres pour, ensuite, formaliser le système de relations spécifique au champ de la probation française ${ }^{21}$.

- La rationalité pénale, au sens pur, se caractérise par sa dimension politique et symbolique, sa finalité étant le paiement d'une dette contractée par un citoyen du fait de la désobéissance aux règles auxquelles il est tenu en tant que citoyen. Cela implique de lui infliger une punition dont la dimension afflictive, quelle qu'en soit la forme, assure la rétribution (paiement) de cette dette. Celui qui applique une telle rationalité est mis dans la position du juge ou de l'homme qui applique la loi. L'objet pénal est mis dans la position du citoyen puni, à la fois confirmé dans sa citoyenneté, dégradé par sa désobéissance mais en voie de réhabilitation grâce à la peine elle-même. Ce qu'il faut retenir ici, c'est que le pénal constitue toujours un socle de légitimation politique, contractualiste, puissant. Pourtant, il a nécessairement dû s'adjoindre d'autres dimensions hétérogènes pour justifier le « vilain métier de punir 22 ».

- La rationalité éducative, qui s'enracine dans la vieille fonction d'amendement, vise précisément un objectif de transformation morale du sujet. La peine ne doit pas uniquement être vengeresse mais aussi servir à l'émancipation par un travail en profondeur de l'individu sur lui-même, travail de transformation des valeurs qui structure sa vision du monde et guide ses comportements - ce que l'on pourrait nommer une « conversion axiologique 23 ». Pour obtenir ce déplacement, il faut établir une relation avec la personne, nécessairement basée sur la confiance pour mettre en discussion ce qui est de l'ordre de la conscience intime. L'éducateur est donc un accompagnateur (e-ducere, conduire vers...), dont l'empathie est requise en tant que condition d'une juste distance face à l'autre. Enfin, l'éduqué est nécessairement mis dans une position de minorité, toute prétention éducative supposant chez lui un déficit d'autonomie et de responsabilité. L'important est ici de percevoir l'effet de légitimation d'une posture normative qui s'ancre en même temps sur un souci pour la dimension subjective de l'individu puni.

- La rationalité sociale a pour finalité la réinsertion des personnes condamnées. Cela suppose une action de « reconstruction » du lien social tournée vers

21. Il s'agit ici de définitions minimales, de schèmes construits à partir d'une analyse des discours structurants du champ de la probation française. Elles s'appuient sur deux sources de discours. D’une part, des textes de référence sur le sujet : normes législatives et réglementaires, prescriptions relatives à la formation des agents, brochures officielles, rapports de diverses provenances, ouvrages théoriques, etc. D'autre part, une série d'entretiens $(n=38)$ réalisés avec des personnels pénitentiaires d'insertion et de probation entre novembre 2011 et janvier 2013, principalement au sein de cinq SPIP (sièges ou antennes) français.

22. Foucault M., Les anormanx. Cours an Collège de France. 1974-1975, Paris, Gallimard/Le Seuil, Hautes Études, 1999, p. 22.

23. Casadamont G., "Institution judiciaire, travail éducatif et inscription sociale », in Études (1983-1987), Plessis-le-Comte, Enap, 1987, pp. 21-35. 
le logement, l'emploi, la formation, l'accès aux droits, les relations sociales et familiales, etc. Le moyen impliqué est double : d'une part l'élaboration d'un projet d'insertion viable en fonction des capacités de la personne mais aussi des contraintes pénales et, d'autre part, la connexion de ce projet avec des possibilités de réalisation sociale, en particulier grâce à l'orientation vers des partenaires (services publics, entreprises privées, associations etc.). Le CPIP est alors en position de conseiller (pour le projet) coordonnateur (vers les partenaires). Ceci implique une relation de proximité avec la personne, le projet requérant la compréhension globale d'une situation et d'un parcours, et une forme de technicité dans la gestion du réseau de partenaires. La personne à insérer est, par définition, conçue négativement par un déficit de statut social qu'elle a le devoir de combler par le travail de Sisyphe d'une insertion interminable ${ }^{24}$. Cette dimension sociale « compense » la dimension strictement rétributive par une justification plus « solidariste » : si le condamné doit « payer » politiquement, il doit aussi être « aidé » socialement.

- La rationalité sanitaire - dont le point de référence est ici celui de la santé mentale - est complexe à saisir, notamment parce que les personnels pénitentiaires ne sont pas censés prendre eux-mêmes en charge les dimensions sanitaires de la peine. On peut retenir comme définition consensuelle de la finalité sanitaire la notion de soulagement d'une souffrance, dans l'horizon d'une santé conçue de manière extensive en tant qu'état de "bien-être » ${ }^{25}$. Son moyen spécifique est le diagnostic et le traitement du trouble qui cause cette souffrance. Or si les CPIP ont a priori une position d'orientation des personnes vers les partenaires de santé, ils sont en fait amenés à occuper une position bien plus ambiguë face à cette question du soin. En effet, ils doivent opérer des sortes de pré-diagnostics pour réaliser ces orientations et juger de la pertinence des mesures d'obligation de soins. Mais il doivent aussi travailler en entretien la question de l'adhésion aux soins pénalement contraints voire, de plus en plus, œuvrer en «collaboration » avec les soignants pour susciter cette « demande » et favoriser ainsi l'ouverture d'un travail thérapeutique. Ceci les place bien dans une position de soin, c'est-à-dire a minima de souci pour la souffrance de l'autre; cet autre étant défini par la «maladie » dont il est essentiellement victime. Cette logique proliférante dans les prises en charge de probation s'appuie, en même temps qu'elle la stimule, sur une posture humaniste qui justifie la pratique par la volonté de soulager autrui.

- La rationalité de gestion des risques criminels affiche quant à elle une prétention techno-scientifique de connaissance et de maîtrise du phénomène criminel, communément appelée « nouvelle pénologie 26 » dans le monde anglo-

24. Castel R., Les métamorphoses de la question sociale, Paris, Gallimard, Folio essais, 1999 [1995], pp. 418-435.

25. Plan Psycbiatrie et Santé mentale 2011-2015, Paris, Ministère du Travail, de l'Emploi et de la Santé, Ministère des Solidarités et de la Cohésion sociale, février 2012, p. 17.

26. Cauchie J.-F., Chantraine G., « De l'usage du risque dans le gouvernement du crime. 
saxon. Selon un vieux schéma d'opposition, la finalité n'est plus ici rétributive - tournée vers l'acte passé - mais préventive - tournée vers la potentialité d'un acte futur. Les moyens de cette gestion sont l'évaluation et le traitement du risque, où l'enjeu n'est plus d'inférer une dangerosité des individus à partir de théories déterministes plus ou moins consistantes, mais de mesurer une probabilité à partir de critères objectivés (physiques, psychiques, comportementaux, sociaux, etc.) et traités statistiquement. Il s'agit ensuite de la prétention à réduire ce risque par des programmes d'action standardisés, soumis à une évaluation quantitative de leur efficacité. L'agent de probation est alors mis dans la position d'un technicien-expert du risque, dont le but est de catégoriser la personne suivie en fonction des facteurs de risque qu'elle incarne. Il faut remarquer la légitimation scientiste et techniciste qui est ici recherchée dans l'espoir, d'une part de consolider un champ professionnel, d'autre part de convaincre les administrateurs, les politiques, voire l'opinion publique de l'efficience du système judiciaire en matière de sécurité publique. Cette rationalité de gestion des risques criminels, est finalement celle qui peut donner un contenu adéquat et précis au vocable flottant de « crimino-logie ». Il faut en effet refuser la dissémination de ce terme, qui peut aussi bien désigner l'évaluation actuarielle du risque ${ }^{27}$ que le travail éducatif et humaniste dans l'application des peines ${ }^{28}$, ou encore la réflexion critique sur le crime comme production sociale ${ }^{29}$. Il s'agit là, en fait, de savoirs hétérogènes sur le phénomène criminel, dont les paradigmes sont incommensurables et les objectifs inconciliables.

- On perçoit immédiatement l'alliance objective qui se noue entre cette gestion des risques et la rationalité de nouvelle gestion publique indexée sur la recherche de l'efficience maximale des systèmes gouvernementaux. Cette recherche d'efficience passe principalement par la quantification et la standardisation de l'action qui mettent l'agent dans une position de comptable. Comptable des ressources dépensées et comptable au sens de la responsabilité vis-à-vis d'une obligation de résultat qui ne dit pas encore son nom, en particulier en termes de responsabilité d'une récidive. La notion de qualité du «service public pénitentiaire » pose le condamné dans la position immédiate-

Nouveau prudentialisme et nouvelle pénologie ", Champ pénal/Penal Field [En ligne], vol. II, 2005 ; Slingeneyer T., «La nouvelle pénologie, une grille d'analyse des transformations des discours, des techniques et des objectifs dans la pénalité ", Champ pénal/Penal field [En ligne], vol. IV, 2007.

27. Ainsi du modèle « RBR », pour Risques, Besoins et Réceptivité, systématisé au Canada et en expansion dans le monde. Sur ce modèle : Bonta J., Andrews A., Modèle d'évaluation et de réadaptation des délinquants fondé sur les principes du risque, des besoins et de la réceptivité, Rapport pour spécialistes n²007-06, Ottawa, Sécurité Publique Canada, 2007.

28. Voir par exemple McNeill F., “Desistance, 'What works?' and community sanctions”, in Mbanzoulou P, Herzog-Evans M., Courtine S., Insertion et désistance des personnes placées sous main de justice, Paris, L'Harmattan, Champ pénitentiaire, 2012, pp. 155-165.

29. Sur la distinction entre une crimino-logie et un champ de savoir sur le crime comme "activité spécifique de connaissance » voir Kaminski D., Mary P. et Cartuyvels Y., « L’autonomie épistémologique de la criminologie : illusoire et inutile », Cabiers Français, La Documentation Française, 372, 2012, pp. 76-79. 
ment paradoxale d'être un usager, à la fois citoyen puni et «client » d'une prestation. D'où une nouvelle forme de légitimation par l'instrumentalisation de l'action publique impliquant une dépolitisation des enjeux jadis politiques de la peine ${ }^{30}$.

\section{Les relations entre rationalités : synergies, contradictions et torsions de sens}

Ces éléments de définitions ne doivent pas être pris pour des réifications de logiques qui, en fait, sont toujours déjà enchevêtrées dans les discours et les pratiques. Ils sont méthodologiques et instrumentaux, ne servant qu'à clarifier les types de relation qui structurent le système de rationalités, afin de les problématiser. Si l'on accepte notre schématisation, trois types de relation sont alors repérables.

Il y a d'abord des formes d'affinités entre rationalités. Ces affinités peuvent être locales, telles que l'alliance objective entre des rationalités gestionnaires (du risque et des ressources) ou l'affinité humaniste du socio-éducatif mais aussi du sanitaire. On peut aussi, et surtout, être attentif aux « synergies » plus transversales, souvent postulées par l'institution lorsqu'elle vante par exemple les mérites de la «pluridisciplinarité » et du «partenariat »; synergies présupposant que la connexion de ces logiques est à la fois cohérente et source d'efficacité dans les prises en charge, à l'instar de la promotion de la « prévention de la récidive » comme l'alpha et l'oméga des pratiques de probation.

Pourtant, ces affinités et ces synergies provoquent inévitablement de multiples contradictions ou hiatus entre des rationalités incommensurables. Le pénal entre ainsi en conflit avec l'humanisme impliqué par les prises en charge : extériorité judiciaire versus relation empathique éducative, logique rétributive versus logique d'aide sociale, dimension afflictive de la peine versus souci sanitaire de soulagement d'une souffrance, etc. De même, les logiques gestionnaires entrent en conflit avec les exigences symboliques de l'application stricte de la justice, comme avec les soubassements axiologiques du métier. La suspicion, la focalisation sur le passage à l'acte voire la quantification du risque impliquées par la rationalité criminologique percutent la confiance, la prise en charge globale tournée vers les déficits de l’individu d'une logique socio-éducative. Le " pragmatisme », le rationnement, la standardisation de la rationalité gestionnaire heurtent l'exigence axiologique, la notion d'obligation de moyens et la prise en compte de la singularité d'une approche plus humaniste et solidaire.

30. Lascoumes P., Le Galès P. (eds.), Gouverner par les instruments, Paris, Presses de Sciences Po, 2004 ; Mary P., « Le Travail d'intérêt général et la médiation pénale face à la crise de l'état social : dépolitisation de la question criminelle et pénalisation du social » in Travail d'intérêt général et médiation pénale : Socialisation du pénal ou pénalisation du social ?, École des Sciences criminologiques Léon Cornil, Bruxelles, Bruylant, 1997. 
Enfin, d'une manière plus subtile, l'articulation (simultanée ou successive) entre ces logiques provoque des torsions de sens inévitables sur les notions manipulées et les registres d'intervention impliqués. Ainsi, dans l'activité d'évaluation et de traitement des personnes suivies, tout se passe comme si l'on postulait la cumulativité des connaissances et la coordination harmonieuse des actions. Le mélange d'informations issues du parcours pénal, de la trajectoire personnelle, du comportement en détention, des attitudes dans les entretiens, de la situation sociale, du contexte affectif, des « diagnostics» de troubles plus ou moins précis (trouble de la personnalité, addiction, perversion...), mais aussi du jugement professionnel tout à fait variable dans sa qualité et ses présupposés selon les différents intervenants, etc., tout cela est censé prendre sens par la magie d'une richesse pluridisciplinaire incontestée. De même, les différents types d'action sur la personne tels que le rappel à la loi, le travail de prise de conscience, la verbalisation et les décharges d'affect, les efforts d'insertion, le traitement des troubles psychiques, la menace de l'incarcération, l'auto-contrôle comportemental, etc., tout cela est pensé comme allant naturellement d'un point $\mathrm{A}$ (l'état délinquant) à un point $\mathrm{B}$ (la sortie de la délinquance). Or, dans le cas de connaissances dont les présupposés sont loin d'être directement compatibles comme dans le cas d'actions dont les effets n'ont aucune raison de s'aligner, le plus probable n'est pas la synergie mais la cacophonie ou le mouvement brownien. Il faut également insister sur le fait que dans l'articulation ou la succession de ces logiques dans les prises en charge, les positions de sujet et d'objet ne peuvent pas se superposer d'une manière rationnelle : il n'est pas possible, logiquement, d'être à la fois un juge impartial, un accompagnateur empathique, un orienteur bienveillant, un soignant, un technicien du risque et un agent du service public face à son usager, même si la situation pratique réalise de fait cette impossibilité. De même qu'être traité comme un citoyen à punir, un immature à éduquer, un exclu à insérer, un malade à guérir, un dangereux à neutraliser, un usager à gérer, cela fait beaucoup pour un seul homme. Pour autant, on repère aisément que ces "problèmes » logiques renforcent en même temps les effets de pouvoir d'un tel système, car si l'on peut demander raison des décisions et de la légitimité d'un pouvoir reposant sur une rationalité positive, il est impossible de le faire devant une rationalité multiforme du pouvoir dont l'éclectisme produit un arbitraire aussi efficient qu'insaisissable.

\section{Le rôle fantomatique d'une rationalité criminologique}

Cette analyse en termes de système de rationalités permet déjà de saisir que le problème actuel n'est pas tout à fait celui de la colonisation du pénal et du pénitentiaire par un savoir ou des pratiques dites « criminologiques». Le problème est bien davantage celui de la place que prend une telle logique dans un système multiple et, surtout, les effets du fonctionnement de l'ensemble du système. Plus précisément, qu'en est-il de la rationalité criminologique, au sens de gestion des risques criminels, dans la probation française ? Comment 
s'insère-t-elle dans ce champ où elle ne représente qu'un élément parmi d'autres ? À la question naïve de savoir s'il existe en France des dispositifs concrets d'évaluation et de traitement des risques dans le champ de la probation, la réponse est clairement non. Les deux dispositifs récents qui ont pu laisser croire au développement d'une telle logique - le diagnostic à visée criminologique (DAVC) et les programmes de prévention de la récidive (PPR) - témoignent en fait de la place ambiguë d'une gestion des risques en France.

Le DAVC, initialement présenté comme un outil d'évaluation du risque de récidive, est rapidement devenu un dossier informatisé témoignant de la situation de la personne à un moment donné. Il s'agit d'un onglet de l'application informatisée APPI (pour Application des Peines, Probation et Insertion) qui comprend des informations sur la situation pénale et le respect des obligations, sur l'appropriation de la condamnation et la reconnaissance de l'acte commis, sur l'inscription dans l'environnement social, professionnel et familial, sur les capacités au changement, sur la prise en charge médicale dans le cadre des mesures privatives ou restrictives de liberté. Pour chaque partie du DAVC, le CPIP doit dégager des axes de travail argumentés et, à la fin, il synthétise la situation et propose des objectifs hiérarchisés pour la prise en charge. Le DAVC initial doit être rempli trois mois après la saisine du SPIP et il est obligatoirement validé par un cadre du service, ce qui déclenche une transmission immédiate à l'autorité judiciaire. D'une manière tout à fait symptomatique, la circulaire du 8 novembre 2011 relative à ce «diagnostic » n'utilise le mot «criminologique » qu'une seule fois (en dehors du C de DAVC), à la toute fin : «Une fois ce travail effectué, le CPIP est en mesure de proposer des modalités de suivi adaptées au profil criminologique de la personne suivie ». Or, cette prétention est tout fait intenable. Il faudrait en effet que de tels profils préexistent au diagnostic (ou que celui-ci serve à les produire). Il faudrait que la série d'informations récoltées soit traitée comme autant de signes dont des configurations précises seraient reliées à un niveau de risque précis. Ce qui produirait des catégories de risque auxquelles on pourrait rattacher chaque personne évaluée. Ce n'est absolument pas le cas ici, ni d'une manière strictement quantitative (des items cotés donnant un score de risque), ni d'une manière plus qualitative. Il n'y a tout simplement aucun lien dans cet outil entre les informations, leur organisation et la mesure d'un risque. Chaque diagnostic n'est finalement que le dossier unique d'une personne ne le rattachant à aucune catégorie; ni profil, ni criminologique, donc. Par ailleurs, pour que cet outil corresponde à une rationalité de gestion du risque, il faudrait que le résultat du diagnostic oriente automatiquement vers des programmes d'action adaptés au profil de risque, visant à faire baisser ce risque et pouvant objectiver leur efficacité. Là encore, rien de tout cela. La proposition de travail du CPIP n'est pas orientée vers la baisse d'un risque (ni par l'outil, ni par la culture professionnelle) mais vers la résolution de problèmes inhérents à la situation de la personne, gênant le respect des obligations pénales, mais aussi sa réinsertion sociale. 
De même, les PPR sont présentés dans la circulaire du 19 mars 2008 comme un mode de prise en charge collectif (sous la forme d'un groupe de parole d'une dizaine de séances animé par un binôme de CPIP), spécifiquement ancré dans la dimension «criminologique » de la mission de «prévention de la récidive ». Ceci impliquerait donc d'orienter résolument la prise en charge sur le passage à l'acte, ses déterminants (i.e. les facteurs de risque de récidive), et ses conséquences sur la victime et la société. La pédagogie privilégiée y serait « d'inspiration cognitivo-comportementale ", visant à modifier les schémas cognitifs jugés inadaptés et corriger les comportements délictueux par l'apprentissage de stratégies d'évitement des situations à risque ${ }^{31}$. Tout porte ainsi à croire que ces programmes s'inscriraient dans une rationalité de gestion des risques criminels puisque, strictement focalisés sur l'acte et ses conséquences, ils ont pour objectif la réduction du risque en misant sur la capacité des individus à s'auto-contrôler. Pourtant, la définition institutionnelle du dispositif de même que son usage par les professionnels invitent à nuancer fortement ce postulat. Du point de vue de sa définition, le PPR semble en effet sans cesse renvoyé à une articulation impossible entre les rationalités éducative et criminologique. C'est ainsi, par exemple, que le Référentiel $\mathrm{PPR}$ peut à la fois énoncer qu' " afin de garantir le caractère criminologique ou éducatif du PPR, l'animation par un binôme psychologue/personnel d'insertion ou l'intervention de tout autre partenaire extérieur ne doit pas être envisagée 32 » puis, quelques pages plus loin, qu' « afin de garantir le caractère “criminologique" et éducatif du PPR, l'animation relève de la compétence exclusive des personnels d'insertion et de probation 33 ». Que penser de la coexistence de ces deux modes opposés de rapport entre l'éducatif « et/ou » le criminologique ? Quelle articulation envisager à partir de l'alternance discrète entre un « et » et un « ou », qui rend indécidable le paradigme propre du dispositif ? Or, cette ambiguïté ne manque pas de faire retour dans les pratiques ellesmêmes. En premier lieu, l'orientation des personnes dans un PPR se fait beaucoup moins en fonction d'un risque perçu (à défaut d'être évalué) qu'en fonction de l'opportunité que ce mode de prise en charge représente au regard de la situation de la personne comme du déroulement du suivi (le passage en groupe étant souvent une réponse à certains freins rencontrés dans le suivi individuel). En second lieu, un consensus semble régner : le PPR est presque unanimement qualifié d'éducatif tandis que le criminologique brille par une quasi absence. Plus précisément, les éléments de rationalité criminologiques du PPR dans sa définition " théorique »sont fréquemment recodés par les professionnels dans les termes d'une rationalité éducative. Le « travail sur le passage à l'acte » fait alors l'objet de discours contrastés, où une compréhen-

31. Pour une présentation institutionnelle du dispositif, voir : Brillet E., « Une nouvelle méthode d'intervention auprès des personnes placées sous main de justice : les programmes de prévention de la récidive (PPR) ", Cabiers d'études pénitentiaires et criminologiques, 31, août 2009; Référentiel Programme de prévention de la récidive, Paris, Direction de l'administration pénitentiaire, mai 2010.

32. Référentiel Programme de prévention de la récidive, op. cit., p. 20. Nous soulignons.

33. Ibid., p. 44. Nous soulignons. 
sion criminologique le dispute clairement à une compréhension éducative. Repérage des distorsions cognitives, analyse de la chaîne délictuelle, apprentissage des stratégies d'évitement des situations à risque, etc. - ou - Réflexion sur le parcours de vie, introspections sur le sens des valeurs d'un monde vécu, implications des choix existentiels, etc. : le PPR se caractérise moins par son ancrage dans une prétendue dimension criminologique que par l'indétermination de sa visée.

Pour autant, il serait naif de considérer que le C de DAVC, ou les notions «criminologiques » des PPR ne sont que des erreurs ou de simples effets de communication. La rationalité de gestion des risques, si elle ne structure pas ces dispositifs concrets, produit des effets importants à d'autres niveaux. Tout d'abord, il y a effectivement des mesures indexées sur la dangerosité des individus, c'est clairement le cas pour la rétention, la surveillance de sûreté et la surveillance judiciaire qui s'ajoutent à la peine sur la base d'une évaluation de dangerosité (reposant essentiellement sur l'expertise psychiatrique pénale). Mais cette manière de voir est également présente dans le suivi socio-judiciaire, de plus en plus au niveau des libérations conditionnelles et, finalement, elle « hante » l'ensemble des pratiques en introduisant la grille de lecture du risque de récidive là où elle n'a par ailleurs souvent pas de sens pour les professionnels.

De ce fait, la notion de " prévention de la récidive » comprise selon le prisme de la gestion des risques (ce qui n’est pas une nécessité) tend à infléchir et subordonner le sens des différents registres d'action - pénal, éducatif, social, sanitaire. Et la subtilité réside en ceci qu'elle le fait moins au niveau de la matérialité même des dispositifs que de leur usage. Par exemple : continuer à rechercher une " prise de conscience » par un accompagnement éducatif, mais en se focalisant d'une manière plus ou moins maîtrisée sur le passage à l'acte ; continuer à œuvrer à l'insertion des personnes, mais en se limitant à ce que l'on perçoit comme en lien avec le risque de récidive ; rechercher une adhésion au soin avec en tête le rapport supposé entre un trouble flou et une dangerosité qui l'est tout autant...

Or, cette diffusion insidieuse d'un registre criminologique sert également à légitimer l'action pénitentiaire sous un angle "pragmatique ». Cela peut être vrai du professionnel arborant une action de «prévention de la récidive » pour pouvoir continuer à agir selon ses propres valeurs; mais aussi du service ou de l'administration qui doivent rendre compte de son activité selon le prisme gestionnaire ; ou encore d'une rhétorique politicienne affichant la prétention d'une efficience préventive en termes de sécurité publique. Mais, en retour, le remplissage de la coquille vide criminologique par des logiques d'action plus réalistes et plus valorisées dans le contexte français, en tout cas professionnellement, permet aussi de légitimer une logique de prévention de la récidive par une sorte de « sublimation » par les valeurs. Bref, une figure tout à fait foucal- 
dienne de la double légitimation : une prétention techno-scientifique concrètement et symboliquement intenable, qui à la fois légitime et est légitimée par des pratiques axiologiques qu'elle juge obsolètes.

\section{Conclusion : Légitimations éclectiques et arbitraires du droit de punir}

Pour autant, telle qu'elle se déploie à partir de l'expertise psychiatrique pénale, par exemple au début du cours au Collège de France de 1974-1975 et culminant dans les considérations sur la naissance d'un pouvoir de normalisation à la pliure entre le pouvoir juridico-politique de la loi et le pouvoir médical de la norme ${ }^{34}$, la critique foucaldienne de la « criminologie » pourrait bien être aujourd'hui retournée contre elle-même. Malgré les doutes que l'on peut leur opposer, les tenants d'une prétention techno-scientifique de la gestion du crime pourraient sans difficulté tourner cette critique à leur avantage en pointant que le domaine de la criminologie est effectivement né dans un intervalle, au croisement de deux formes de pouvoir et de savoir consistantes selon leurs critères intrinsèques. Le jeu entre la rationalité judiciaire (reposant sur la triade infraction-culpabilité-rétribution selon un triple registre juridiquemoral-politique) et la rationalité médicale (reposant sur la triade maladiesouffrance-traitement selon un triple registre normatif-humaniste-technique) aurait alors produit un troisième terme, un nouveau champ d'investigation possédant ses propres catégories : la déviance aux normes collectives en tant qu'elle représente un danger qu'une nouvelle forme de "pouvoir-savoir » technique viendrait circonscrire, prévenir et neutraliser. Certes, nous disent les «criminologues », ce champ, par définition plus « jeune » que les deux autres, ne possède pas encore la consistance épistémologique et politique de ses aînés mais il ne cesserait de témoigner de ses progrès dans la quête de son autonomie, jusqu'au moment fantasmé où il pourra, enfin, «tuer » le père, et la mère ! Et, de fait, il n'est pas si facile de balayer d'un revers de la main la construction d'un système de justice très intégré basé sur une rationalité de gestion des risques, comme c'est le cas au Canada en particulier. Un tel système n'incarnerait-il pas le produit d'un dépassement tant espéré de l'opposition loi-norme (ou Justice-Santé), vis-à-vis duquel la France accuserait simplement, mais résolument, un retard certain ?

Tout se passe comme si une lecture réifiante et dualiste des rationalités judiciaire et médicale permettait une interprétation quasi « odipienne » d'émancipation du fils maudit. C'est pourquoi nous avons préféré suivre ici les parti-pris méthodologiques de la discursivité et de la multiplicité. Tout d'abord, la réification du couple « pouvoir-savoir » que représenteraient la justice et la médecine autour de processus historiques génétiques et d’institu-

34. Outre les développements des cours successifs, on en trouve des formulations claires dans de nombreux entretiens tout au long des années 1970. Par exemple : Foucault M., «L'extension sociale de la norme », in Foucault M., Dits et écrits, Tome III, Paris, Gallimard, 1994 [1976], pp. 74-79. 
tions situées peut avoir pour effet d' « attester » la réalité de l'autonomisation d'un champ criminologique. La mise en œuvre concrète de cette rationalité dans des dispositifs de gouvernement permet de masquer, sous l'effet d'une « évidence » pragmatique, les contradictions logiques qu'ils impliquent pourtant. À l'inverse, le niveau d'analyse discursif met l'accent sur les différences irréductibles entre les rationalités qui trament les pratiques, en lieu et place de l'unification «pragmatique » des lignes synergiques que promeut, entre autres, le fonctionnement institutionnel. En France, une telle unification est surtout rhétorique et passe par la notion de «prévention de la récidive » qui peut s'interpréter dans les termes de chacune des rationalités en présence (pénale, éducative, sociale, sanitaire, gestionnaire) tout en favorisant, d'une manière insidieuse, le prisme de la gestion du risque. Insister sur la cohérence interne minimale des différentes formations discursives qui orientent et légitiment les pratiques, et donc sur leurs contradictions, c'est immédiatement problématiser l'efficacité de cette orientation et la validité de cette légitimation ${ }^{35}$.

Ensuite, la grille de lecture dualiste justice-médecine nourrit l'illusion d'un possible dépassement « dialectique » de l'opposition originaire alors qu'il faut, à l'inverse, penser l'accumulation, comme telle problématique, des formes de rationalité. Si l'on peut isoler une rationalité « criminologique ", en aucun cas elle ne remplace, ni même n'englobe, les autres formes de rationalité avec lesquelles elle compose. Au contraire, l'analyse discursive permet de saisir que le problème est moins la colonisation du champ pénal par un « troisième terme » criminologique incarnant une nouvelle forme hégémonique de pouvoir et un nouveau grand discours de légitimation, que la multiplicité des rationalités et des « jeux de langage » qui structurent et légitiment ce champ. La question de la légitimité du droit de punir ne se pose dès lors plus au niveau superficiel d'une «vraie » ou d'une «fausse » science criminologique, mais au niveau structural du croisement des logiques éclectiques, incommensurables et dissensuelles, du champ pénal. Or, s'il est possible de penser la criminologie au point de croisement des deux grands discours de légitimation par la loi et par la science, il n'est pas possible de penser, donc de localiser, le point d'intersection entre le pénal, l'éducatif, le social, le sanitaire, le criminologique et le gestionnaire. Le cœur du problème n'est pas la criminologie mais l'éclectisme pénal qui permet de «fonder » un droit de punir d'autant plus redoutable que son « socle » est impossible à situer, au croisement impensable de logiques incompatibles.

35. D'ailleurs, contrairement à ce que l'on peut souvent entendre, de telles ambiguités ont aussi été repérées comme structurelles dans le système canadien : "Cette situation ambiguë, au sein de laquelle s'affrontent l'idée d'un traitement et d'une relation d'aide individualisée d'un côté, et une préoccupation croissante ou même dominante pour la gestion informatisée de risques statistiques de l'autre, n'est pas sans leur poser de nombreux problèmes ». In Vacheret M., Dozois J., Lemire G., « Le système correctionnel canadien et la nouvelle pénologie : la notion de risque », Déviance et société, 22-1, 1998, pp. 37-50, p. 44 